\title{
LUGAR Y FUNCIÓN DE LOS ATLETAS OLÍMPICOS EN LA FILOSOFÍA DEL BARÓN PIERRE DE COUBERTIN ${ }^{1}$
}

\author{
Clément Anicet GUIYAMA-MASSOGO \\ Presidente de la Academia Olímpica de la República Centroafricana \\ Presidente de la Asociación Francófona de las Academias Nacionales Olímpicas, \\ Director del Programa CAMS del Comité Olímpico Internacional. \\ guimassogo@yahoo.fr \\ Fecha de recepción: mayo 2018 \\ Fecha de aceptación: octubre 2018
}

DOI: http://doi.org/10.15366/citius2018.11.2.001

Resumen:

En este trabajo el autor analiza las bases del ideario del Barón Pierre de Coubertin en su conjunto, exponiendo y fundamentando la mayor relevancia que otorgó a los atletas y a los roles que debían desempeñar. En su planteamiento, las acciones del atleta se relacionaban con acciones excepcionales que debían tener un impacto positivo en el funcionamiento global de la sociedad. También resume ciertas acciones llevadas a cabo por la estructura del Movimiento Olímpico Moderno y el Comité Olímpico Internacional (COI) para situar a los atletas en el corazón del Movimiento Olímpico como la Agenda 2020.

Palabras clave: Rol del atleta, Ideario Olímpico, Pierre de Coubertin, Movimiento Olímpico, atleta, olimpismo.

\section{Title: PLACE AND FUNCTION OF THE OLYMPIC ATHLETES IN THE PHILOSOPHY OF BARON PIERRE DE COUBERTIN}

\begin{abstract}
:
In this work, the author analyses the basis of the ideology of Baron Pierre de Coubertin as a whole, exposing and basing the greater importance he gave to athletes and the roles they should play. In his approach, the actions of the athlete were related to exceptional actions that should have a positive impact on the overall functioning of society. It also summarizes certain actions carried out by the structure of the Modern Olympic Movement and the International Olympic Committee (IOC) to place athletes at the heart of the Olympic Movement as the Agenda 2020.
\end{abstract}

Key words: Role of the athlete, Olympic Ideology, Pierre de Coubertin, Olympic Movement, athlete, Olympism.

\section{Introducción}

Es un gran honor para mí recibir la invitación del Presidente de la Academia Olímpica Internacional y presentar en esta sesión «El papel de los atletas olímpicos en la filosofía del Barón Pierre de Coubertin» (esperando haber traducido bien en francés el espíritu y el título de la comunicación que se me propuso en inglés). También me alegro de poder compartir con mis compañeros de los Comités Olímpicos Nacionales y de las Academias Olímpicas mis

\footnotetext{
${ }^{1}$ El presente trabajo corresponde al contenido de la conferencia presentada por el autor en la Decimocuarta Sesión Internacional de Presidentes o Directores de las Academias Olímpicas Nacionales y equipos de los Comités Olímpicos Nacionales, celebrada en Olimpia (Grecia), entre el 6-13 Mayo 2018. Este artículo supone la versión en español preparada especialmente para la edición en el presente número de Citius, Altius, Fortius. Humanismo, Sociedad y Deporte.
} 
conocimientos sobre el tema tratado, y aprovecho para agradecer a la dirección de la Academia Olimpica Internacional la oportunidad que me ha brindado.

Desde hace décadas, la Academia Olímpica Internacional realiza un trabajo excepcional en lo que se refiere a la difusión de los ideales olímpicos. Estos ideales han sido trasmitidos en todas las partes del mundo, por un lado, por las Academias Olímpicas Nacionales, los Centros o Institutos de Estudios Olímpicos y, por otro lado, por las Asociaciones de las Academias Olímpicas Nacionales, que incluyen la Asociación de Academias Nacionales Olímpicas de África (AANOA) y la Asociación Francófona de Academias Olímpicas (AFOA) de las que formo parte. Por lo tanto, es un deber para mí destacar las buenas relaciones de colaboración que se están forjando entre la Academia Olímpica Internacional y las asociaciones, y que justifican además mi invitación a esta sesión, pues asisto en representación de la Asociación Francófona de Academias Olímpicas. Sin ninguna duda, estas estrategias de acciones entrecruzadas entre las estructuras de difusión de la filosofía olímpica sólo pueden beneficiar a la causa olímpica que defendemos, pues justamente esta estrategia se basa en la complementariedad de funciones y estructuras y no sobre planteamientos opuestos $\mathrm{e}$ injustificados.

Como testigo y actor en el seno de las sesiones de la Academia Olímpica Internacional desde hace más de dos décadas, he podido constatar que varios de los temas abordados por los conferenciantes a lo largo de la historia de esta institución, han girado en torno al papel del atleta olímpico. En consecuencia, baso mi presentación en el legado de la reflexión siempre presente en nuestra Academia Internacional, proporcionando una visión renovada de los problemas tratados en los últimos años en el seno del Movimiento Olímpico Internacional.

Si efectivamente el tema que voy a tratar es recurrente en nuestro ámbito, es seguramente por una buena razón, ya que no es el atleta, sino el joven que practica deporte, quien constituye la esencia de nuestro movimiento. De tal manera que, aunque existe el deporte para todos, y que éste es cada vez más popular en todo el mundo, son en realidad los Juegos Olímpicos, como todas las grandes competiciones internacionales, los que más movilizan a los jóvenes que practican deporte y que buscan un reconocimiento a través de la obtención de medallas.

\section{El atleta en el planteamiento de Pierre de Coubertin}

Con una visión muy acertada, Pierre de Coubertin concibió los juegos como el festival de la juventud mundial, como el rencuentro de atletas de todo el mundo, simbolizado a través de los cinco anillos de la bandera olímpica. En calidad de renovador de los Juegos Olímpicos, así como de fundador del Comité Olímpico Internacional, el Barón Pierre de Coubertin situó a los atletas en el centro del Movimiento Olímpico. El actual presidente del COI, Thomas Bach afirmó recientemente que «los atletas son el corazón de los Juegos Olímpicos» (discurso de presentación de la Agenda 2020 en Mónaco en 2014). Considerando esta filosofía, podemos comprender el papel asignado a los atletas tanto en el seno del movimiento deportivo como en la sociedad.

¿Por qué deberíamos prestar más atención a los atletas dentro del Movimiento Olímpico y en qué medida la figura del Barón Pierre de Coubertin estableció las bases para otorgar mayor relevancia a los atletas y a los roles que debían desempeñar? Tal es la complejidad de este doble planteamiento que, para intentar clarificarlo, he recurrido a la Carta Olímpica y a las recomendaciones de la Agenda 2020 del COI. Evidentemente, tengo que ceñirme al tema que se me ha encomendado, pero será bajo la filosofía del Barón Pierre de Coubertin que trataré de esclarecer las cuestiones planteadas.

En una primera aproximación a las obras del Barón Pierre de Coubertin se vislumbra una mayor preocupación por los deportes, la renovación de los Juegos Olímpicos, y la 
consolidación y notoriedad del COI después de su creación. Las numerosas cartas escritas por el Barón, sus viajes y las reuniones que organizó, así como los actos de organización de Los Juegos, justifican el sentido y el espíritu de su obra. Esta interpretación puede, además, estar legítimamente ligada a las Memorias Olímpicas en las que relata los grandes esfuerzos personales que debió hacer en la promoción del deporte y los juegos olímpicos, así como en la creación del COI y en el fortalecimiento de su figura en el plano internacional.

Esta interpretación, como sabemos sin ninguna duda, ha motivado la idea de que Pierre de Coubertin no habría desarrollado una filosofía si se hubiera centrado en la figura del atleta de manera aislada. Este planteamiento no concuerda ni con el espíritu ni con la carta de su filosofía, por lo que deducimos que tenía un nuevo planteamiento muy bien definido.

En sus Memorias Olímpicas, siempre se refiere explícitamente a una «doctrina olímpica integral» $\gg^{2}$ lo que significa que había vislumbrado una concepción del deporte y del olimpismo de carácter sistemático. Esta idea de deporte también fue destacada por Yves Pierre de Boulogne en su obra, al confrontar esta filosofía con los problemas de su época y especialmente, con los problemas de la sociedad francesa del siglo XIX.

Por lo tanto, podemos interpretar más correctamente su obra según el modelo de los tres círculos concéntricos que consiste en: un núcleo, un vivero y una fachada. Este modelo, concierne explícitamente a los miembros del COI y al modo de funcionamiento de la institución, tal y como el Barón lo había pensado e implementado con el fin de garantizar su sostenibilidad. Sin embargo, tengo la certeza de que este modelo determina y se aplica a toda la filosofía olímpica del Barón. En consecuencia, el núcleo es el atleta, el adolescente o el deportista, el vivero es la pedagogía o la educación combinada con la cultura francesa, y la fachada se entiende como el cambio social considerado como la perfección intelectual y moral de la humanidad. A todo ello se le ha denominado sincretismo coubertiniano que combina la cultura o religión, la filosofía política y un sistema filosófico nuevo, lo que puede justificar la amplia interpretación de la obra.

Es bien sabido que la obra de Coubertin es una exaltación de la belleza del «Kourus», pues asumía que las acciones del atleta se relacionaban con acciones excepcionales que debían tener un impacto positivo en el funcionamiento global de la sociedad, un ejemplo son los dioses de la Grecia Antigua en honor a los cuales se organizaban los juegos. Yves Pierre Boulongne afirma en este sentido: «el atleta coubertiniano, es la actualización de Ganimedes, el más bello de los hombres mortales según Homero» ${ }^{3}$.

El COI tiene como función principal educar a los jóvenes a través del deporte con el fin de promover los valores olímpicos en la sociedad, pues es el joven atleta olímpico el que constituye el corazón del movimiento impulsado en el siglo XIX por el Barón Pierre de Coubertin. Por lo tanto, para lograr la preparación de este joven atleta para la vida adulta, fue necesario definir el concepto de «vivero». Para el Barón Pierre de Coubertin es la pedagogía o la educación olímpica la que empieza a vincular la educación con los valores olímpicos.

Este concepto se presenta para el Barón como el medio más seguro para valorar de una manera racional la juventud, amenazada por el desorden, la inseguridad e insidias de todo tipo. Además, la pedagogía fue percibida como un cambio social. En su Campaña de 21 años, habla del mejor y más eficaz instrumento:

«Sigo convencido que, tanto en 1908 como en 1887, la pedagogía deportiva, tal y como Thomas Arnold la comprendía, es la mejor y más eficaz herramienta en la que pueden apoyarse todos los

\footnotetext{
${ }^{2}$ Y.P Boulongne, Pierre de Coubertin, Humanisme et pédagogie. Dix leçons sur l'olympisme, Documents du Musée du CIO, Lausanne, 1989.

${ }^{3}$ Y.P Boulongne, Pierre de Coubertin, Humanisme et pédagogie. Dix leçons sur l'olympisme, Documents du Musée du CIO, Lausanne, 1989.
} 
educadores de todos los países, con el fin de formar adolescentes tanto desde el punto de vista moral como físico» ${ }^{4}$.

Tanto como pedagogo, como en su rol de teórico de la historia olímpica, el Barón Pierre de Coubertin comprendió la importancia y el interés que la formación y el aprendizaje adquirían especialmente en los jóvenes o adolescentes, para su inserción social. En su visión del deporte, unida a las experiencias vividas en Inglaterra, la pedagogía se convirtió en una fuente de gran utilidad para conseguir ciudadanos excepcionales, capaces de luchar contra sus debilidades, así como contra las formas alienantes de la sociedad. Esta visión le llevará a dar un lugar prominente a las universidades en su trabajo de renovación de los deportes antiguos respaldando la convicción de que «el deporte sin consciencia es solamente una ruina para el hombre» ${ }^{5}$.

El vivero sobre el modelo cultural es la base o el terreno a partir del cual las plantas germinan y pueden crecer. Esta metáfora sugiere que el vivero, además de ser el canal por el que las plantas se desarrollan, es también la base de la germinación o del desarrollo. Por lo tanto, la pedagogía coubertiniana no es solo la base, sino el crisol de la germinación de espíritus jóvenes para el deporte. Tal es así, que su lema era «el espíritu en la escuela de los deportes» ${ }^{6}$.

No obstante, para comprender mejor el papel fundamental de la pedagogía, es importante ponerlo en consonancia con el objetivo que le había sido asignado en la obra del Barón, así como con el contexto sociopolítico de su época.

Además, conviene recordar que el Barón Pierre de Coubertin se encontraba a caballo entre dos épocas y dos sistemas de gobierno diferentes: la aristocracia con sus valores, y la república naciente también con sus valores propios. En contra de todo pronóstico, se propuso realizar una síntesis de los valores subyacentes a los dos sistemas de gobierno con el fin de crear un nuevo mundo orientado hacia el progreso en todos los campos.

Coubertin, como buen estratega, concibió ese mundo nuevo como la fachada de su obra, es decir, como la fachada visible del edificio, pero que es solamente comprendido en estrecha relación con el núcleo, es decir, con aquello que gira en torno al edificio. El ideal del nuevo y mejor mundo que él formuló y que se ha establecido como el objetivo del Olimpismo Moderno en la Carta Olímpica, no es factible, aparte del atleta olímpico entrenado de alguna manera para lograr acciones excepcionales como hemos mencionado anteriormente. En el marco de la simbiosis entre los valores aristocráticos y los valores republicanos que quiso integrar, su acción educativa era considerada como la contribución del deporte y, por ende, del atleta, al desarrollo liberal de la sociedad, al tiempo que integraba la excelencia aristocrática. Él mismo expresó esta idea en los siguientes términos:

«El deporte en Francia, será intelectual o no será. Será el fiel y discreto compañero de la reflexión, del ideal, de la imaginación; será el baluarte silencioso y bien guardado detrás del cual el individuo realizará su crecimiento intelectual» ${ }^{7}$.

Por otro lado, desde el punto de vista de nuestro autor, el crecimiento intelectual es inseparable del ascenso social en la medida en la que el perfeccionamiento del individuo está ligado a su responsabilidad de buen ciudadano, o incluso al perfeccionamiento moral de la humanidad. También y, en definitiva, a través del deporte y especialmente del atleta olímpico, Coubertin aspiró a la creación de esa super humanidad descrita por Nietzsche, que estableció ciertos hitos y que sigue siendo objeto de búsqueda dentro del Movimiento Olímpico.

\footnotetext{
${ }^{4}$ Pierre de Coubertin, Campagne de vingt-et-un ans, p.206.

${ }^{5}$ Y.P Boulongne, Op. cit., p 70.

${ }^{6}$ Baron Pierre de Coubertin, Mémoires Olympiques, p.85.

${ }^{7}$ Baron Pierre de Coubertin, Op. ci.t, p.73
} 
En la actualidad, seguimos insistiendo de una manera más incisiva, en el reforzamiento del rol del atleta en el seno del Movimiento Olímpico como consecuencia de los numerosos debates sobre su figura. ${ }^{8}$ Un claro ejemplo fue la octava conferencia mundial sobre Deporte, Educación y Cultura en noviembre de 2012 en Ámsterdam. El tema principal de esta conferencia fue «La juventud y la educación: motor del olimpismo», y éste constituyó uno de los temas de esos debates e intercambios. Por otra parte, se han multiplicado a escala mundial las iniciativas que permiten que los atletas expresen sus principales preocupaciones, así como las reflexiones sobre el rol que están llamados a desempeñar. ${ }^{9}$

Se considera efectivamente que los atletas y, por tanto, los jóvenes, tienen una posición destacada en el Movimiento Olímpico. El antiguo presidente del COI y exatleta olímpico, el doctor Jaqcues Rogge afirmaba: «...el Movimiento Olímpico del mañana pertenece a los jóvenes de hoy... si los jóvenes aprenden a respetarse en las pistas, serán capaces de observar esas actitudes en otras circunstancias de su vida cotidiana» ${ }^{10}$. De manera más precisa, el Boletín N. ${ }^{\circ} 9$ del COI de enero de 2008 proporciona una definición actualizada del papel del atleta contemporáneo:

«La condición de atleta de hoy supera considerablemente los límites de las pistas. El atleta debe tener voz durante y después de su carrera deportiva. Debe poner al servicio del deporte y de la sociedad, su pasión, su experiencia y sus competencias. Es esencial que sea capaz de tener una influencia en el desarrollo del deporte, así como en la toma de decisiones dentro de las organizaciones deportivas». ${ }^{11}$

Si tomamos al pie de la letra el acta, el COI tiene ahora un dispositivo completo destinado a afirmar y consolidar el lugar nodal del atleta en el Movimiento, lo que supone escuchar y tener en cuenta sus deseos. Es por tanto una postura, que asocia directamente al atleta con el movimiento y que le implica a todos los niveles importantes de gestión del deporte y de las competiciones deportivas.

Este planteamiento que se ha ido concretando con el paso del tiempo, ha adquirido otra dimensión desde 2014 con la adopción de la hoja de ruta del Movimiento Olímpico denominada Agenda 2020, basada en la credibilidad y la buena gobernanza en el deporte. El informe anual del COI 2015 titulado «Credibilidad, Durabilidad y Juventud», no solamente plantea un ejemplo de buena gestión del órgano supremo del Movimiento, sino que también expone las acciones realizadas por el COI durante el año, basándose en las cuatro principales corrientes entre las que se encuentra justamente «situar a los atletas en el corazón del Movimiento Olímpico».

Sin duda, sería interesante desplegar todo este dispositivo para hacer un buen contacto con las evoluciones en cuanto al estatuto y el papel de los atletas en el Movimiento Olímpico Internacional hasta nuestros días. Por otro lado, aunque me dirijo a un público que trata a diario de promover la figura del atleta olímpico, creo que es importante destacar el papel innovador de la Agenda 2020, en la que al menos 5 de las 40 recomendaciones conciernen específicamente a la condición del atleta contemporáneo. Se trata de un concepto del atleta íntegro en el que la protección es esencial y para la que es necesario un presupuesto de 20 millones de dólares.

No nos equivocamos cuando afirmamos que se trata de un paso importante en la mejora de la condición del atleta contemporáneo siempre sensible a ciertas lacras del deporte, tales

\footnotetext{
${ }^{8}$ Los informes de las diversas conferencias, reuniones y simposios organizados sobre este tema hacen mención de ello.

${ }^{9}$ El Boletín de Información de la Comisión de Atletas del COI recoge todas la iniciativas y acciones que se llevan a cabo.

${ }^{10}$ Dr Jacques Rogge, Manuel Enseigner les valeurs, avant propos, 2007.

11 Georges Weah en Liberia, que fue recibido recientemente por el presidente francés Emmanuel Macron y que formuló el proyecto de una plataforma de desarrollo de infraestructura deportiva en África
} 
como: dopaje, trampas, engaños y comercialización abusiva. En este sentido, se trata también de volver al ideal de Coubertin del «Kouros», que implica deshacerse de la escoria que va en contra de los valores y que aún sigue minando el deporte. La vuelta al ideal coubertiniano se basa en la figura del atleta como defensor íntegro de la probidad del deporte y constituye, además, un referente en la lucha contra los conflictos sociales, y por tanto, en la lucha contra el odio entre naciones. El mismo Barón ya planteó esta reflexión durante su mensaje de radio en Berlín en 1935.

Podrían rebatirme que esto es solo un ideal y que la naturaleza humana será siempre necia, tal y como apuntaba Voltaire. En relación con los jóvenes, por ejemplo, ¿no es verdad que en ciertas partes del mundo son, desafortunadamente, una poderosa armada de destrucción masiva?

Reconociendo esta realidad indiscutible, yo persisto en creer en una fuerza de cambio positivo inherente en la filosofía olímpica del Barón Pierre de Coubertin, presentada aquí esquemáticamente bajo la forma de los círculos concéntricos y que, en el fondo, no era más que una estrategia de pacificación de las sociedades humanas. La antigua directora general de la UNESCO, Irina Bokova, afirmaba en la octava conferencia mundial de Deporte, Educación y Cultura de 2012:

«Gracias al deporte, podemos promover el cambio ahí donde otros no llegan, convenciendo al público no solamente del inmenso potencial del deporte sino también del lugar natural que ocupan los jóvenes en todo este proceso».

Irina Bokova, fue una voz autorizada por las Naciones Unidas y que constituía el reconocimiento de la fuerza de la pedagogía deportiva, entendido como uno de los pilares del Barón Pierre de Coubertin. Desde entonces, se ha impuesto una cooperación creciente entre el COI y los organismos ligados a las Naciones Unidas, especialmente en relación con el concepto de deporte al servicio del desarrollo y de la paz. Este sistema de cooperación está adquiriendo una mayor dimensión e intensidad, acelerando así el proceso de construcción de un mundo mejor y más pacífico.

La Asociación de Academias Nacionales Olímpicas de África, por un lado, y la Asociación Francófona de las Academias Olímpicas, por otro, han tomado consciencia de este desafío y de los retos que representan esos conceptos. En este sentido, dichas Academias se ocupan de desarrollar acciones concretas de intensificación de la oferta olímpica a favor de la paz y del desarrollo, tal y como se expondrá en su presentación. Solo queda una dinámica de carácter global que habrá que implantar con el apoyo del COI, así como con los colaboradores internacionales, especialmente la UNESCO. Hay por tanto que esperar a que un día todas las instituciones encargadas de la divulgación y de la difusión de la filosofía olímpica en el mundo, se sienten en una mesa para definir estrategias conjuntas destinadas a promover la cultura olímpica en todo el mundo. Gracias.

\section{Bibliografía}

\subsection{Referencias bibliograáficas}

- Bailey, S. (2008). Athlete first. A History of the Paralympic Movement. Londres: Wiley and Sons.

- Boulongne, Y. P. (1989). Pierre de Coubertin, Humanisme et pédagogie. Dix leçons sur l'olympisme, Documents du Musée du CIO, Lausanne.

- Boulongne, Y. P. (1992) : La vie et l'œuvre pédagogique de Pierre de Coubertin, Leméac. 
- CIO. (15 de septiembre de 2017). Charte Olympique du CIO. Laussane: COI.

- CIO. (2013). Héritage Olympique. Laussane: COI

- CIO. (2016). Code d'Ethique. Laussane: COI

- Coubertin, P. (1921). Leçons de pédagogie sportive. Lausanne.

- Coubertin, P. (1977). Mémoires Olympiques. Publications du CIO, Lausanne.

- During, B. (1989). Valeurs de l'olympisme. INSEP, Paris.

- Eyquem, M.T. (1966). Pierre de Coubertin, l'épopée olympique. Calmann Lévy, Paris.

- Guiyama-Massogo, C. A. (2003). L'olympisme comme philosophie de la paix. Dossier de recherche. Centre d'Etudes Olympiques, Lausanne.

- Guiyama-Massogo, C. A. (2009). An analysis of traditional sporting practices and the dissemination of olympism in Africa based on study of case of Central African Republic (La connaissance des pratiques sportives traditionnelles et la diffusion de l'olympisme en Afrique Centrale à partir du cas de la République Centrafricaine) (en anglais et en chinois), Olympic Studies Reader. Beijing Sport University.

- Guiyama-Massogo, C. A. (2011). Réflexions sur un système de gestion rationnel des organes dirigeants du sport en Centrafrique: le cas du Comité National Olympique, mémoire de master professionnel en management des organisations sportives (MEMOS) du Comité International olympique, Université de Poitiers.

- Guiyama-Massogo, C. A. (2015). Sport, santé et développement en République Centrafricaine, Annales de l'Université de Bangui, série A, 11(2), Décembre.

- Mzali, M. (1996). L'olympisme aujourd'hui. Paris : Ed. Jeune Afrique.

- Tribou, G. (2016). Sponsoring sportif, $5^{\mathrm{e}}$ edition. Economica.

\subsection{Otras publicaciones y consultas web}

- Bulletin d'information de la Commission des Athlètes du CIO, diversos numéros.

- Bulletins du Centre d'Etudes Olympiques de Lausanne (Suisse).

- Revue Olympique, différents numéros. Lausanne (Suisse).

- Rapports sur les conférences mondiales sur le sport et l'environnement.

- Rapports sur les Fora sur le sport au service de la paix et du développement.

- Rapports sur les conférences sur le sport, l’Education et la Culture.

- Rapports de la conférence mondiale de l'AMA pour la lutte contre le dopage dans le sport.

- News letter du Centre d'Etudes Olympiques.

- Rapports annuels du CIO 2015, 2016 et 2017. 
- http://www.olympic.org/documents/commissions

- http://www.samsung.com/fr/aboutsamsung/citizenship/hopeforyoung.html

- AIO. (Octubre de 2015). Journal officiel de L'AIO (l'Agenda Olympique 2020). 